\title{
Personalized Multi-Period Tour Recommendations
}

\begin{abstract}
During a trip planning, tourists gather information from different sources, select and rank the places to visit according to their personal interests, and try to devise daily tours among them. This paper addresses the complex selection and touring problem and proposes a "filter-first, tour-second" framework for generating personalized tour recommendations for tourists based on information from social media and other online data sources. Collaborative filtering is applied to identify a subset of optional points of interest that maximize the potential satisfaction, while there are some preselected mandatory points that the tourists must visit. Next, the underlying orienteering problem is solved via an Iterated Tabu Search algorithm. The goal is to generate tours that contain all mandatory points and maximize the total score collected from the optional points visited daily, taking into account different day availabilities and opening hours, limitations on the tour lengths, budgets and other restrictions. Computational experiments on benchmark datasets indicate that the proposed touring algorithm is very competitive. Furthermore, the proposed framework has been evaluated on data collected from Foursquare. The results show the practical utility and the temporal efficacy of the recommended tours.
\end{abstract}

Keywords: Orienteering Problem, Iterated Tabu Search, Tour Recommendations, Collaborative Filtering

\section{Introduction}

Trip planning is a complex and time-consuming process (Souffriau et al., 2013). Tourists need to gather information from paper-based and online data sources (e.g., travel guides, web sites, blogs etc) and make a selection of the points of interest to visit that correspond most to the their personal interests. They shortlist and prioritize the points, while iteratively they try to design the tours and determine the visiting sequences subject to a set of hard and soft constraints. Apparently, numerous alternatives may emerge and besides 
the selection of points, the touring problem is computationally intractable and hard to solve. It typically maps to orienteering problems, in which the underlying mathematical models take into account a wide variety of restrictions and satisfaction metrics (Souffriau et al., 2008, Vansteenwegen et al., 2011a, Zhu et al., 2012). This paper presents a novel "filter-first, routesecond" framework that captures the tourist's preferences based on social filtering as well as it generates high-quality personalized tour recommendations considering various intuitive constraints and realities.

It is evident that tourists increasingly abandon standard tours in favor of more personalized options (Hyde et al., 2003). Paper-based travel guides provide only generic recommendations that cannot meet the specific preferences of every tourist. On the contrary, one of the recently introduced functionality of digital travel guides is personalized tour recommendations (Gavalas et al., 2014, Anacleto et al., 2014). Notably, the users of these digital tools do not only have access to the opinion and viewpoint of an expert but they can also obtain access to the opinion of their peers through text reviews. Furthermore, the content is shared with social media users and online reviewers, who can then continuously evaluate its quality and make sure it is up-to-date.

Despite recent advances, the vast majority of the existing expert or automated tour planning systems are still inflexible in the sense that they provide the user with a set of pre-computed tours instead of personalized recommendations based on given set of preferences and constraints. For instance, many of the systems proposed in the literature do not consider the category of the points to be recommended or the user's preferences with respect to each category (e.g., De Choudhury et al. (2010), Roy et al. (2011), Dunstall et al. (2003), Kim et al. (2009)). Furthermore, the recommendations made often ignore the visiting horizon, the tour lengths and the traveling distances, and the inter-dependencies between the consecutive daily tours. Our framework addresses all these issues in a number of ways and introduces a very rich formulation for addressing the touring problem that, to the best of our knowledge, appears for the first time in the literature.

The contributions of this paper can be summarized as follows. First, we propose a collaborative filtering (CF)(Deshpande and Karypis, 2004). Given a set of mandatory points selected by the tourist this technique estimates preferences and recommends an additional set of optional points that are expected to maximize satisfaction. We acknowledge that it is challenging for a tourist (with limited knowledge of the currently popular points of interest 
and attractions in the city) to select the complete subset of places to visit that will maximize the utility of the tour. Instead, the tourist's input is much more likely to be limited to a small set of mandatory points, chosen based on personal interests and word-of-mouth. Second, we develop a network-based integer programming mathematical model to capture the multi-day touring problem. The aim is to decide, for each day of the planning horizon, the mix of optional and mandatory points, as well as to determine the visiting sequence that maximizes the utility of each daily tour. Our formulation takes into account temporal constraints (e.g., multiple time windows, maximum tour lengths and travel time constraints) as well as other user-defined restrictions including budget restrictions for entrance or other type of fees and category quotas (e.g. a maximum number of museums to visit each day). The objective is to maximize the total collected from the visited optional points. The resulting model can be abbreviated as the Multi-Period Multiconstraint Orienteering Problem with Multiple Time Windows (MP-MC-OPMTW) and it is solved via an Iterated Tabu Search (ITS) metaheuristic algorithm. Our approach is capable of producing high-quality heuristic solutions in very short computational times.

We assess the performance of the proposed models and algorithms through extensive computational experiments using both artificial and real-life data collected from Foursquare for four main US cities, i.e., New York City, Pittsburgh, Boston and San Francisco. We found that our framework generated multi-day tours with short waiting times, high collected scores and high utilization levels. Notably, compared to the existing orienteering problem literature, our model formulation is more flexible and provides a unique combination of characteristics. To our knowledge, the only model in the literature with multiple periods and multiple time windows is that of Tricoire et al. (2010). For this reason, we used their datasets to benchmark our metaheuristic algorithm and the results verify its competitiveness.

The remainder of the paper is organized as follows. Section 2 comments on the existing literature. Section 3 provides a formal description of the multi-period personalized tour design problem. Section 4 presents the proposed framework and describes in detail all major algorithmic components. Computational experiments assessing the value of the proposed framework, along with a comparative performance analysis, are presented in Section 5 . Next, Section 6 discusses how the proposed framework can be implemented in ways that enhance visitor satisfaction and enable destinations to better promote their tourism resources. Conclusions are drawn in Section 7. Fi- 
nally, note that for the purpose of brevity we will primarily use the term point and interchangeably with the terms location, event, venue, activity, attraction and point of interest.

\section{Related Work}

Research on trip planning and personalized tourist guides have recently attracted significant attention. The so-called tourist trip design problem has been initially introduced by Vansteenwegen et al. (2007). In this seminal work the Orienteering Problem (OP) is used as the basis for modeling the single daily tours. Each attraction, event, activity or point of interest in a city is associated with a profit (or score). The pairwise travel times between the points are known, and the main restriction is that the total tour duration should not exceed a predefined time budget. The objective is to design the tour so that the collected profit or the number of visited points is maximized (Vansteenwegen et al., 2011b, Gunawan et al., 2016).

During the last decade, the above archetypal OP model has been enhanced in various ways to capture multiple user constraints. Vansteenwegen et al. (2007) and Gavalas et al. (2014) discuss the following problem characteristics and constraints: (i) planning tours for multiple days; (ii) grouping the points under different categories and limiting the number of categories to be visited during a tour; (iii) grouping the points as mandatory and optional with the aim to cover all the mandatory points; (iv) hard and soft time windows (e.g. opening and closing hours, admission hours, weather dependencies, accessibility restrictions for disabled tourists); (v) multiple time windows for selected points during the same day or during the planning horizon; (vi) budget constraints (e.g. entrance fees, tickets, and accommodation fees); and (vii) other preferences (e.g. lunch breaks).

A rich multi-day trip planning model has been recently proposed by Souffriau et al. (2013), namely the Multi-Constraint Team Orienteering Problem with Multiple Time Windows (MC-TOP-MTW). Besides multiple time windows on the same day and daily tour duration restrictions, this model considers that every location is associated with several attributes, and every attribute has a budget that cannot be exceeded. These constraints can be seen either as knapsack or as max-n type constraints. For example, budget limitations for entrance fees and maximum number of art locations to visit on a single day. An Iterated Local Search (ILS) algorithm combined with a

Greedy Randomized Adaptive Search Procedure (GRASP) is developed for 
solving the MC-TOP-MTW. Note that the "teams" allow the modeling of multiple days as well as the modeling of multiple tours for the same day.

Zhu et al. (2012) divide the available points into two groups; accommodation and tourism points, and aim to maximize the profit for both groups, considering budget constraints, hotel stays, number of available days, time limits for each day, time windows (same for all days) and weighted profits as per user inputs. On the other hand, multiple-modes of transportation and time-dependent traveling times are considered by Garcia et al. (2013), while Gavalas et al. (2015) include the constraint of using only available public transport, considering the transportation times and schedules for different modes of public transportation.

Another tour planning problem that recently appeared in the literature is the Orienteering Problem with Hotel Selection (OPHS). Given a set of points with a score, and a set of hotels, the goal is to determine a number of connected tours that visits a subset of the points and maximizes the sum of the collected scores. Each tour is limited in length and should start and end in one of the hotels. Divsalar et al. $(2013,2014)$ propose Variable Neighborhood Search and Memetic algorithms for solving the OPHS.

Other closely related orienteering problems to that considered in this paper are the so-called Orienteering Problem with Time Windows (OPTW) and the Multi-Period Orienteering Problem with Time Windows (MuPOPTW). Iterated Local Search (ILS) approaches for the OPTW appear in the works of Vansteenwegen et al. (2009) and Souffriau et al. (2013). Multi-period orienteering problem settings with single as well as multiple time windows are introduced by Tricoire et al. (2010). Both exact and metaheuristic algorithms are proposed and computational experiments for the OPTW and the MuPOPTW with single and multiple teams are reported. We refer to the survey paper of Gunawan et al. (2016) for a detailed overview of orienteering problem variants, models and solution approaches.

Compared to the above models, the proposed MP-MC-OP-MTW provides a unique combination of features that according to our knowledge appears for the first time in the literature. It considers visit restrictions and priorities (i.e. mandatory and optional points to visit), multiple days, varying daily availabilities for each point, multiple time windows during the same day, multiple max-n type restrictions for each day, and duration and budget constraints. Observe that the MuPOPTW shares many similarities with the MP-MC-OP-MTW without considering any teams. For this reason, the benchmark datasets introduced by Tricoire et al. (2010) are employed in this 
paper as the test bed for evaluating the performance of our touring method.

From the application perspective, applied tour recommendation systems initially appear in the works of Gavalas et al. (2011) and Rodriguez et al. (2012). Specifically, Gavalas et al. (2011) describes a web system for deriving personalized recommendations for daily sightseeing itineraries. Multiple user constraints are taken into account, and the objective is to maximize the collected profit associated with the visited points. The profit of every point depends on its category (e.g. architectural monuments and museums) and on the user's preferences upon this category.

Rodriguez et al. (2012) presents a more interactive system for designing customized tourist trips. Multiple objectives are considered and the most representative solutions are shown to the user. This triggers an interactive process. The user provides information about the preferences for the different solutions shown, and the search for the most suitable solution is repeated, respecting the user preferences and constraints. Apart from the maximization of the utility, the authors also consider the minimization of the distance traveled, the minimization of the costs, and the minimization of the difference between the desired time spent on each type of visit and the real time spent for each type of visit. A two-phase metaheuristic algorithm is adopted to generate "efficient" and "compromise" sets of solutions following a globalcriterion method. The utility is calculated based on the "importance" (e.g. rating offered by tourism promotion media) and the tourist's "preference" (scale rating) with regards to the type of the point (e.g. beaches).

The above works focus more on the tour planning component and give less attention on how to model user preferences and how to assign profits to the points. In that respect, more elaborate systems are presented by Souffriau et al. (2008) and Vansteenwegen et al. (2011b) that use online reviews and other documents to identify different point categories (e.g. art, architecture, religious and nature) and to assign personalized profits for each category. Initially, the system queries the user interests for different point types (e.g. restaurants, museums and churches), and creates a profile based on arbitrary keywords provided by the user (e.g. football, military and literature). Next, scores are calculated using a vector space model. The idea is to match the user profile with the corresponding document vector of each point. The document vectors are generated based on a documents database maintained for each point. Various user constraints are taken into account, while construction heuristic algorithm (similar to Chao et al. (1996)) coupled with local search is employed for solving the resulting tour design problem. 
Another framework that takes advantage of dynamic generated content from multiple tourists has been recently proposed by Brilhante et al. (2015). In particular, a two-step planning approach is presented. At first, an unsupervised method for mining common patterns of movements of tourists is used to determine high interest areas. Next, a variant of the Traveling Salesman Problem is solved in order to generate the sightseeing tours.

Despite recent advances, research on the design of holistic frameworks for generating personalized tour recommendations is still in its infancy. It is unclear how to accurately model preferences, as part of the optimization process, using online data sources and opinion mining tools. In this paper, we propose a framework that combines a sophisticated metaheuristic algorithm to generate tours with a social filtering technique to identify an optional set of promising points to visit based on user preferences, visitation relationships, online reviews and number of check-ins. The distinction between mandatory user selected and optional points translates into an easier selection process for the user, while it also seeks to provide a more personalized tour experience. It also fits well to the situation that the user has limited knowledge about the destination and the current trending venues, and can only provide a small set of core points to be included into the tours.

\section{Multi-Period Personalized Tour Design Problem}

Assume a user (tourist) that travels to a city for $P$ days (periods) with the aim of visiting a number of points every day. For generating the daily tours, two sets of points are considered, namely the mandatory set $(\mathcal{M})$ that consists of the points that the user must visit, and the optional set $(\mathcal{O})$ that consists of the points the user can optionally visit during the $P$ days. The former set is defined by the user and reflects personal preferences, while based on this information, the optional set of promising points to visit is generated using a collaborative filtering technique and various online data sources (see Section 4.1 for more details). Note that while all the mandatory points must be visited within the $P$ days of the planned tour, not all the optional points can be visited.

Let a complete undirected graph $G=(V, A)$, where $V=\{0,1, \ldots, n, n+1\}$ is the set of points (nodes) to be visited, and $A=\{(i, j) \in V \times V: i \neq j\}$ is the arc set. Points 0 and $n+1$ denote the starting and ending points of each daily tour, and the point set excluding the starting and ending points is $V_{c}$ so that $V_{c}=V \backslash\{0, n+1\}$. Each point $i \in V_{c}$ can be either mandatory or 
optional, while $V_{c}=M \cup O$. Note that the starting and ending points may refer to the same location (e.g. a hotel) or different locations (e.g. a park and a restaurant). In the first case, the daily tours can be seen as Hamiltonian cycles in the graph, while in the second case the daily tours refer to Hamiltonian paths that visit each point at most once.

Based on the above representation, various problem features and user constraints can be defined. First, we consider the daily availabilities of the points (e.g. bank holidays, weekends, and memorial days). For this purpose, a binary parameter $\xi_{i p}$ for all $i \in V_{c}$ and $p \in P$ is used to indicate whether point $i$ is available on day $p$. Note that whenever point $i$ is available, i.e., $\xi_{i p}=1$, it has a profit $q_{i}^{p} \in \mathbb{R}_{+}$. This profit can be seen as the utility of visiting this particular point compared to others.

Next, a visiting cost is defined for each point (e.g. entrance fees and food prices) as $m_{i}^{p} \in \mathbb{R}_{+}$. On this basis, the users can define the total budget $F_{p} \in \mathbb{R}_{+}$, that they are willing to spend on day $p$. Another attribute defined for each point is the visiting time and it is denoted as $s_{i}^{p} \in \mathbb{R}_{+}$. The visiting time of a point is the time needed to be spent at that point.

Further, a point can be visited within a single or multiple time intervals (windows) during the day. Points with no time windows are assumed to be available for 24 hours. The available time windows are denoted as $L=$ $\left\{1, \ldots, n_{l}\right\}$ for each day. Note that the time windows can be the same or different for each day, thus the calendar days can be taken into account. Each of the time windows have an opening time $b_{i_{l}}^{p}$, and a closing time $e_{i_{l}}^{p}$, so that the time window $l$ of point $i$ is shown as $W_{i_{l}}^{p}=\left[b_{i_{l}}^{p}, e_{i_{l}}^{p}\right]$. The set of time windows for point $i$ on day $p$ is denoted as $T W_{i}^{p}=\left[W_{i_{1}}^{p}, \ldots, W_{i_{l}}^{p}\right]$. Accordingly, continuous variables $a_{i}^{p}$ indicate the arrival time to $i$ on day $p$, and binary variables $x_{i j}^{p}$ indicate whether $j$ is visited after $i$ on day $p$.

All points are classified into categories, such as museums, restaurants and bars. Let $C$ denote the set of categories as $C=\left\{1, \ldots, n_{c}\right\}$, and let the binary indicator $k_{i c}$ to denote whether point $i$ belongs to category $c \in C$. On this basis, max-n type constraints can be defined, i.e., maximum number of points $\phi_{c}^{p}$ to be included in the daily tour $p$ from category $c$.

Whenever a user traverses the $\operatorname{arc}(i, j) \in A$ incurs a traveling distance (or traveling time) $c_{i j} \in \mathbb{R}_{+}$. We assume that the traveling distance is independent of day $p$, the distances are Euclidean and the cost matrix $\left[c_{i j}\right]$ satisfies the triangle inequality. Lastly, let $D_{p}$ be the maximum total distance (or maximum tour duration) the user is willing to cover on day $p$.

The above described tour design problem corresponds to the MP-MC- 
OP-MTW and can be formulated as a mixed-integer programming model. The objective is to maximize the total collected profit.

$$
\begin{aligned}
& \max \sum_{p=1}^{n_{p}} \sum_{i=0}^{n+1} \sum_{j=0}^{n+1} q_{i} x_{i j}^{p} \\
& \sum_{j=1}^{n+1} x_{0 j}^{p}=\sum_{i=0}^{n} x_{i(n+1)}^{p}=1 \quad \forall p \in P \\
& \sum_{i=0}^{n+1} x_{(n+1) i}^{p}=\sum_{j=0}^{n+1} x_{j 0}^{p}=0 \quad \forall p \in P \\
& \sum_{i=0}^{n} x_{i k}^{p}=\sum_{j=1}^{n+1} x_{k j}^{p} \leq \xi_{k}^{p} \quad \forall p \in P, k \in V_{c} \\
& x_{i j}^{p}+x_{j i}^{p} \leq 1 \quad \forall p \in P, \forall i, j \in V, i \neq j \\
& a_{i}^{p}+x_{i j}^{p}\left(s_{i}^{p}+c_{i j}\right)-\left(1-x_{i j}^{p}\right) D_{p} \leq a_{j}^{p} \quad \forall p \in P, i \in V, j \in V_{c} \\
& b_{i_{l}}^{p}+x_{i j}^{p}\left(s_{i}^{p}+c_{i j}\right)-\left(1-x_{i j}^{p}\right) D_{p} \leq a_{j}^{p} \quad \forall p \in P, \forall l \in L, i \in V, j \in V_{c} \\
& 0 \leq a_{i}^{p}+\xi_{i}^{p}\left(s_{i}^{p}+c_{i N}\right) \leq \xi_{i}^{p} D_{p} \quad \forall p \in P, i \in V_{c} \\
& a_{0}^{p}=0 \quad \forall p \in P \\
& a_{i}^{p} \in\left[b_{i_{l}}^{p}, e_{i_{l}}^{p}\right] \quad \forall p \in P, \forall l \in L, i \in V \\
& \sum_{i=0}^{n} \sum_{j=1}^{n+1}\left(c_{i j}+s_{i}^{p}\right) x_{i j}^{p} \leq D_{p} \quad \forall p \in P \\
& \sum_{i=1}^{n} \sum_{j=1}^{n+1} m_{i}^{p} x_{i j}^{p} \leq F_{p} \quad \forall p \in P, i \neq j \\
& \sum_{p=1}^{n_{p}} \sum_{i=0}^{n} x_{i k}^{p}=\sum_{p=1}^{n_{p}} \sum_{j=1}^{n+1} x_{k j}^{p} \leq 1 \quad \forall k \in V_{c}, k \neq i, k \neq j \\
& \sum_{p=1}^{n_{p}} \sum_{i=0}^{n} x_{i k}^{p}=\sum_{p=1}^{n_{p}} \sum_{j=1}^{n+1} x_{k j}^{p}=1 \quad \forall k \in M \backslash(0, n+1), k \neq i, k \neq j
\end{aligned}
$$




$$
\sum_{i=0}^{n+1} \sum_{j=0}^{n+1} k_{i c} x_{i j}^{p} \leq \phi_{c}^{p} \quad \forall c \in C, \forall p \in P, i \neq j
$$

Constraints (2) and (3) ensure that every tour starts at the same starting point and ends at the same ending point. Constraints (4) ensure that only the available points for each period are taken into consideration, whereas constraints (5) ensure connectivity of the solution and handle symmetrical solutions. Constraints (6), (7) and (8) depict the time stamp of the model. Constraints (9) ensure that each tour starts at time 0 and no waiting is allowed before the starting point. Constraints (10) handle the multiple time windows for each point and ensure that a point can only be visited within one of its multiple time windows. Knapsack like constraints (11) and (12) bound the time and monetary budget for each daily tour. Constraints (13) ensure that all optional points are visited at most once, whereas constraints (14) ensure that all mandatory points are visited. Finally, constraints (15) handle the category quotas (max-n type constraint) for each daily tour.

Indicative daily tours of a 3-day trip are depicted in Figure 1. The objec-

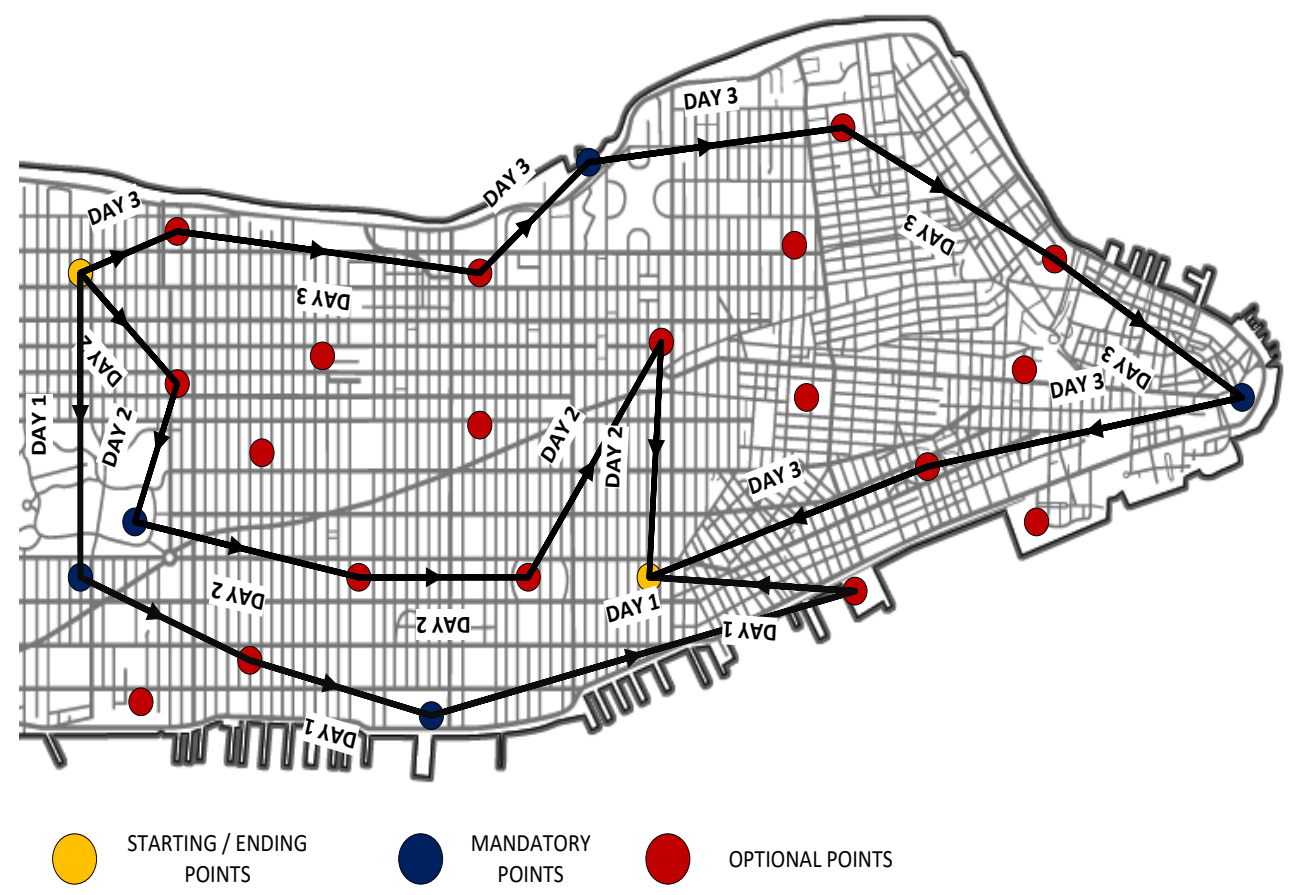

Figure 1: A sample 3-day trip at the lower Manhattan area of NYC 


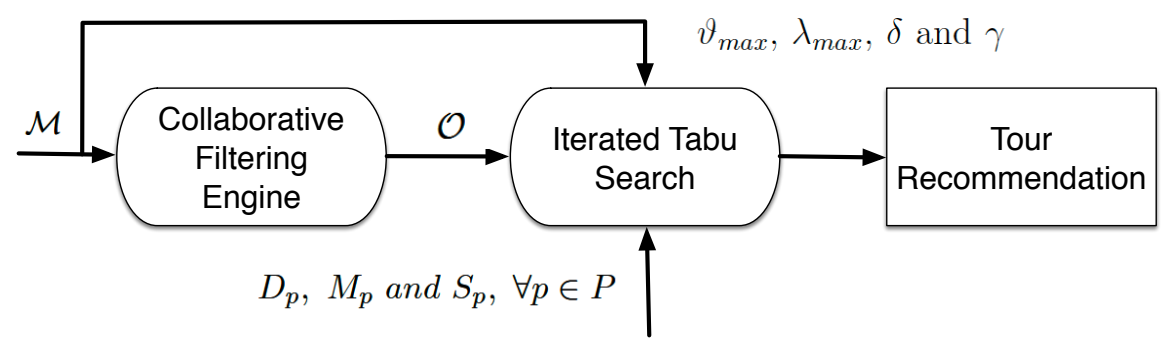

Figure 2: Tour recommendation system - FFTS framework

tive is to maximize the personalized collected profit for every period. This objective is very common in the OP literature and it is also widely adopted in models dealing with tourist trip planning applications. However, other alternative objectives can be also treated with minor changes in the proposed model, such as the maximization of the number of visited points or the minimization of the distance traveled per day (assuming a minimum collected profit as a constraint). Other more advanced multi-objective schemes can be also employed and we refer readers to the work of Rodriguez et al. (2012).

\section{4. "Filter-First, Tour-Second" Framework}

In this section we describe the two main modules of the proposed "FilterFirst, Tour-Second" (FFTS for short) framework. The first module takes as input the set of user-specified mandatory points $\mathcal{M}$ and outputs a set of optional points $\mathcal{O}$ that the user is also likely to enjoy. Then, sets $\mathcal{M}$ and $\mathcal{O}$ together with the user's constraints (e.g. the maximum daily distance $D_{p}$, the daily budget $F_{p}$ and the daily category quotas $\phi_{c}^{p}$ for all days) provide the basic input for the algorithm that solves the touring problem and outputs the final recommendation. The FFTS framework is depicted in Figure 2.

\subsection{Filter-First - Collaborative Filtering}

In order to identify a set of optional points for user $u$ we rely on an itembased Collaborative Filtering (CF) technique for the top- $N$ recommendation problem (Deshpande and Karypis, 2004). In particular, given a user-item 
matrix $\Pi$ that encodes transactions between users and items (e.g., purchase relationships, visitation relationships as in our case etc.) and a set of items $\mathcal{Y}$ that user $u$ has previously selected, the top- $N$ recommendation problem aims at identifying an ordered set of items $\mathcal{X}$ such that $|\mathcal{X}| \leq N$ and $\mathcal{X} \cap \mathcal{Y} \neq \emptyset$.

In our setting, set $\mathcal{Y}$ is the set of mandatory points $\mathcal{M}$ that $u$ wants to visit, while $\mathcal{X}$ is the set $\mathcal{O}$ that we want to identify. Furthermore, the user-item matrix $\Pi$ captures visitation relationships. In particular,

$$
\pi_{i j}= \begin{cases}1, & \text { if user } i \text { has visited point } j \\ 0, & \text { otherwise }\end{cases}
$$

Matrix $\Pi$ will be created using check-in information from Foursquare (details on the dataset are provided in Section 5). The item-based CF builds a similarity model that identifies for every item (i.e., point) the $k$ most similar items. The model is finally represented through a square matrix $\mathbf{F}$, where $F_{i j}$ is the similarity between elements $i$ and $j^{1}$. This similarity between two items can be computed in a variety of ways. For instance, two items $i$ and $j$ can be treated as a vector at the point space (i.e., the $i^{\text {th }}$ and $j^{\text {th }}$ column of $\Pi$ respectively) and the similarity between $i$ and $j$ is simply the cosine between these two vectors. In our work, we use a conditional probability-based similarity definition that has been shown to outperform cosine similarity (Deshpande and Karypis, 2004). Specifically, using the recorded transactions in the useritem matrix, we can calculate the conditional probability $P(j \mid i)$ of selecting (visiting in our case) item $j$, given that $i$ has already been selected. This is simply the ratio between the number of users that have transactions with both items $i$ and $j$, over the total number of users that have transactions with item $i$. Given that $P(j \mid i)$ is not necessarily equal to $P(i \mid j)$, this approach leads to an asymmetric similarity measure that can lead to problematic recommendations. We correct this by dividing with the number of users that have transactions with item $j$. Furthermore, care needs to be taken to avoid possible biases from users that have "purchased" many items. In particular, with $\rho_{i}$ being the number of users that have a transaction with item $i$, we have:

\footnotetext{
${ }^{1}$ Note here that since we are interested in the $k$-nearest neighbors of each item, every row (or column) of $\mathbf{F}$ has only $k$ non-zero elements. The rest are suppressed to 0.
} 


$$
F_{i j}=\frac{\sum_{\forall q: \pi_{q j}>0} \pi_{q j}}{\rho_{i} \cdot \rho_{j}^{\alpha}}
$$

where $\alpha \in[0,1]$. More details can be found in Deshpande and Karypis (2004).

Finally, once the model is built, we can evaluate our query by providing the set of items that our user has already selected. In our setting this is the $\mathcal{M}$ set, which includes points that have been pre-approved by the user. The query is a vector $\mathbf{q}$, where every entry corresponds to a point; $q_{i}=1$ if our user wants to visit point $i$; otherwise, $q_{i}=0$. Then we simply project the query vector to the latent space identified from our model, that is, we compute $\mathbf{x}=\mathbf{F} \cdot \mathbf{q}$. The final recommendations for $\mathcal{O}$ consist of the $N$ elements of $\mathbf{x}$ with the highest value that are not already in $\mathcal{M}$.

\subsection{Tour-Second - Iterated Tabu Search}

Given the sets $\mathcal{M}$ and $\mathcal{O}$, an iterated tabu search algorithm is applied to generate the daily tours. Algorithm 1 depicts the proposed multi-start local search scheme. At first, a greedy randomized construction heuristic is employed (see Line 3) to find a feasible tour for each day that will include all mandatory points as well as a number of optional points, if possible. At this point, the main iterated tabu search component is triggered (see Line 4). The oscillations between the construction heuristic and the local search algorithms are repeated for a number of iterations $\psi_{\max }$ (termination condition), while at the end of this process the best solution found is reported considering as primary objective the collected profit (denoted with $f$ ) and as secondary objective the total distance traveled (denoted with $g$ ). Input parameters $\vartheta_{\max }, \lambda_{\max }, \delta$ and $\gamma$ control the number of internal local search restarts, the termination condition for the local search (number of iterations without observing any improvement), the tabu tenure (tabu list size) and the perturbation mechanism, respectively. The proposed construction heuristic and iterated tabu search algorithms are described in Sections 4.2.1 and 4.2.2.

\subsubsection{Construction heuristic algorithm}

A serial insertion-based greedy construction heuristic algorithm has been developed for producing feasible initial solutions. Our algorithm is based on the earlier work of Chao et al. (1996). Initially, an ellipse is drawn, taking the starting and ending points as the foci points and the maximum allowed 


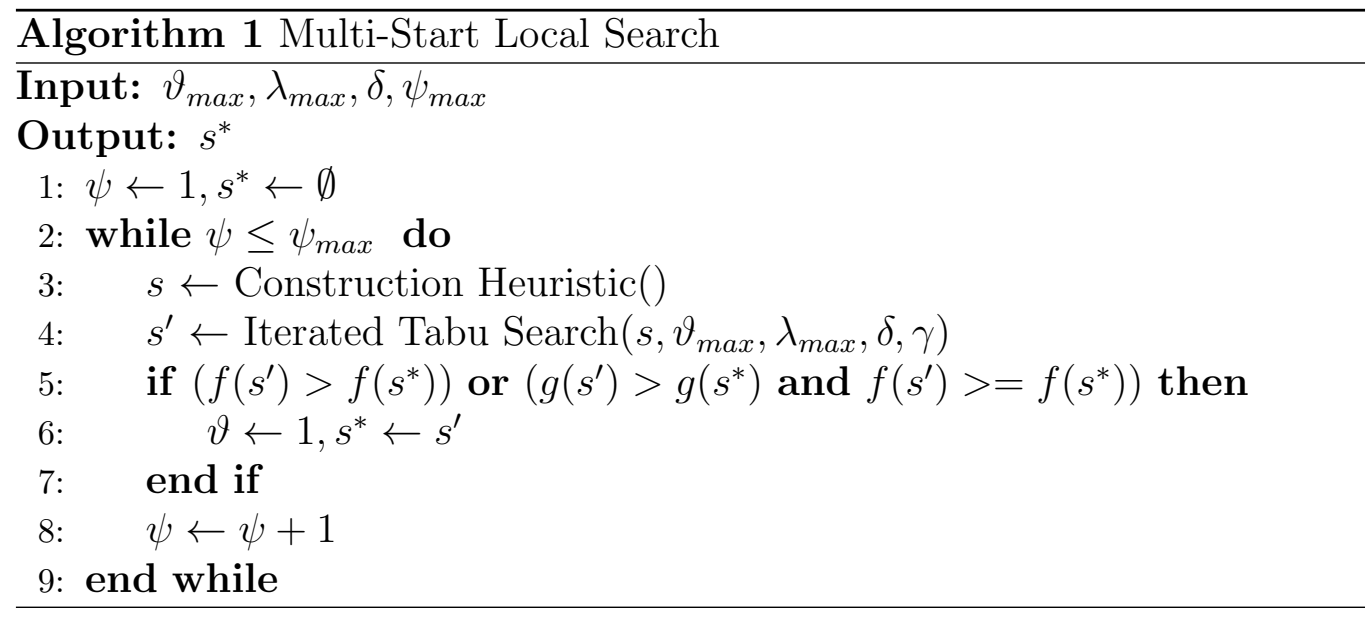

traveling distance as the length of the major axis. Then, the points that are inside the ellipse are identified from the set of all points, in accordance with the general ellipse equation. If all the mandatory points are not inside the ellipse, i.e. the time budget is too low for the construction heuristic to include every mandatory point, the algorithm terminates. The points are identified and prioritized starting from the one that is further away from the foci points of the ellipse. This search also prioritizes the mandatory points, while considering the time windows, monetary budget and category quota restrictions. The increase in profit, cost and solution duration, as well as category information are stored for each added point.

Having identified the available set of points, the tour construction scheme is triggered. At first, all mandatory points are inserted one by one based on a greedy function that takes into account the distance traveled. At each iteration, for all un-inserted mandatory points and for all feasible insertion positions, we calculate how much the distance traveled is increased and we select to insert the point that causes the minimum detour. Next, a similar serial insertion scheme is followed for including optional points. The insertion of optional points is performed considering a weighted combination of the cheapest insertion cost (detour) and the profit of each point, while it continues until the time budget is reached and no other points can be inserted in the tour. Feasibility checks are performed with the insertion of every possible optional point candidate. Note that the weights in the greedy function are randomly selected so as to generate diversified solutions.

It is straightforward to perform feasibility checks for every period regard- 
ing the monetary budget and the category quotas. It is also well known in the literature how to use the so-called push forward calculations in order to perform feasibility checks for time window and maximum route duration constraints. However, in case of multiple time windows these calculations should be modified accordingly. In particular, we need to determine first the arrival time at each point, and based on this to select the time window that each point will be visited. Assuming that the available time windows for each point are not overlapping, we always select the time window with the closest opening to the arrival time. This approach allows feasibility checks to be performed with minimal computation effort. Interested readers may refer to the work of Tricoire et al. (2010) for more general cases and feasibility calculations for problems with multiple time windows.

\subsubsection{Iterated Tabu Search}

Starting from the solutions generated via the construction heuristic, an Iterated Tabu Search algorithm is applied for further improvement. As depicted in Algorithm 2, there are two main components; the tabu search and the perturbation. The tabu search involves the exploration of the solution space by moving at each iteration from a solution $s$ to the best admissible solution $s^{\prime}$ of the neighborhood structure $N_{y}(s)$ according to a tabu list (see Lines 5-15). Specifically, two criteria are used for evaluating the neighboring solutions in a hierarchical fashion. The primary objective is the profit $f$, while the secondary objective is the distance traveled $g$ (see Lines 8 and 10). This hierarchical search direction is motivated from the earlier work of Tarantilis et al. (2013a). The tabu list (short term memory) records the most recently visited solutions and prevents revisiting them for a predefined number of iterations $\delta$ (tabu tenure). The tabu status of a neighboring solution can be overridden, only if predefined aspiration criteria are met. To help the search escape from local optimal solutions, whenever the local search has performed $\lambda_{\max }$ iterations without observing any improvement, the current best solution is perturbed and the local search restarts.

The neighborhood structures $y$ used within the proposed implementation are based on traditional edge-exchange local moves, namely intra- and inter-route 2-Opt, 1-0 Relocate and 1-1 Exchange, modified accordingly for the examined problem. A permutation based representation scheme is followed. The neighborhood structures are randomly selected with equal selection probability. Since the solutions are produced for multiple periods, inter- and intra-period moves are also considered, with the exception of 2- 


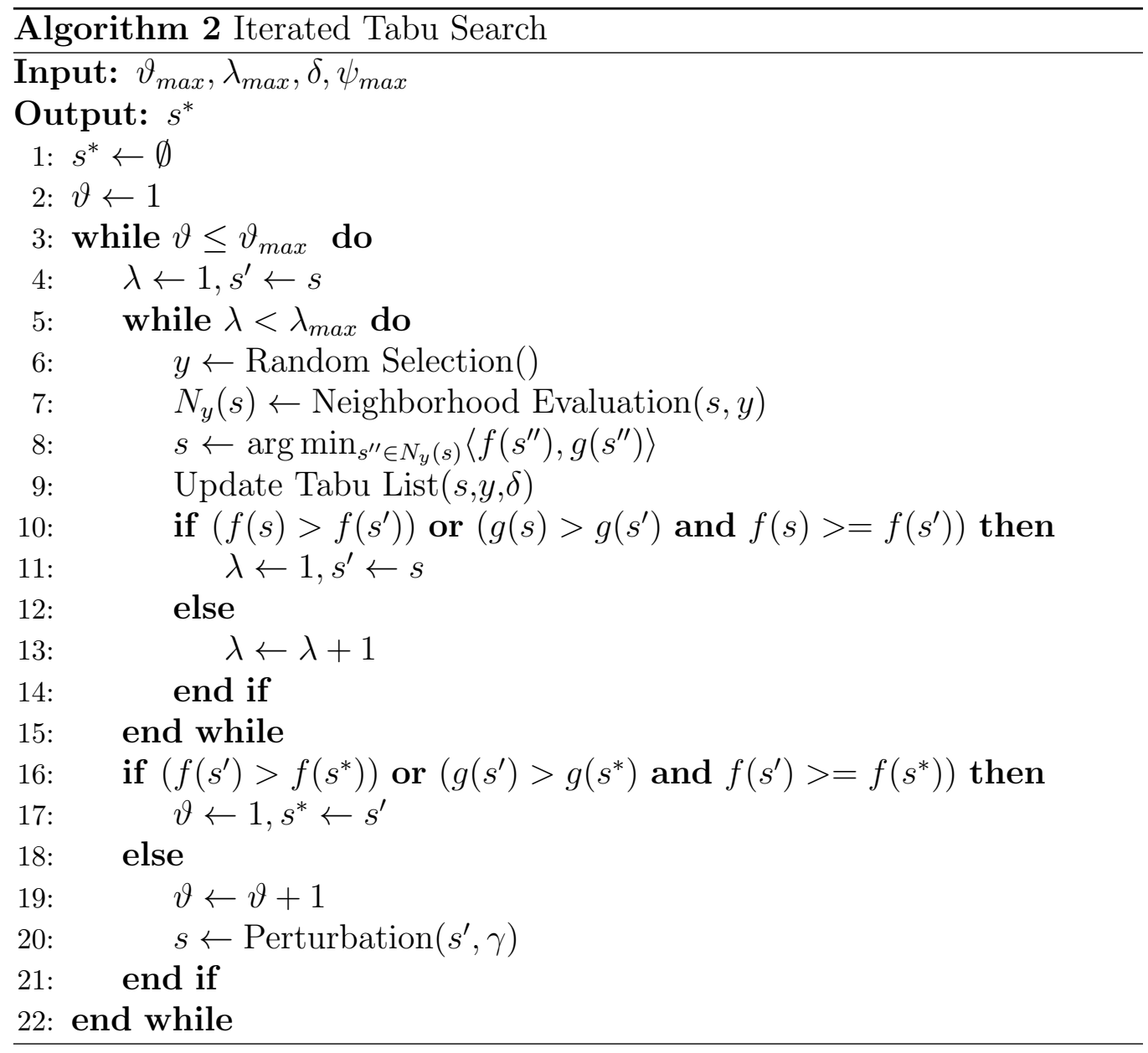

Opt move being only for intra-period. Updating the time stamps of this representation is straightforward, but whenever an inter-period move is applied, the sequence-dependent arrival times on the points must be updated accordingly. To that end, a lexicographic ordering search is followed for evaluating the neighborhood structures using the time window feasibility check procedures proposed in the earlier work of Tarantilis et al. (2013b).

Intra-period 2-Opt reverses the visiting sequence of a segment (series of points visited sequentially) $(i, \ldots, j)$. Next, intra-period 1-0 Relocate and 11 Exchange swap two disjoint segments $(i, \ldots, j)$ and $\left(i^{\prime}, \ldots, j^{\prime}\right)$ that contain 0 and 1 points, respectively. On the other hand, inter-period 1-0 Relocate can involve either the relocation of one point from one period to another or 
the insertion of a currently non-visited optional point to the tour at a given period. For a sample 3-day trip (see Figure 1), examples of intra- and interperiod Relocate moves are shown at Figures 3 and 4 . Changes are depicted with red colored lines. Similarly, inter-period 1-1 Exchange can involve either the swap of two points visited in two different periods or the insertion of a currently non-visited point with simultaneous removal of a visited point at a given period. Examples of intra- and inter-period Exchange moves can be seen at Figures 5 and 6.

To avoid cycling, both the forward and reversal move attributes, i.e., edges being added and deleted, of the corresponding neighborhood structure are stored within the short term memory. In our implementation, the tabu list is maintained at constant size $\delta$, while the tabu status is overridden if an improvement is observed with respect to the best encountered solution. Lastly, at each restart the perturbation mechanism randomly selects and removes a subset of points from every period with a probability $\gamma$.

\section{Computational Experiments}

Various computational experiments have been performed to assess and evaluate the proposed framework. Initially, we test the proposed Iterated Tabu Search algorithm on existing benchmark datasets provided by Tricoire et al. (2010) for the MuPOPTW. Section 5.1 presents the results obtained from our algorithm and compares them with those obtained from the Variable Neighborhood Search algorithm of Tricoire et al. (2010). Next, we generate a real dataset collected from Foursquare to demonstrate the effectiveness of the FFTS framework as a whole for solving a wide set of tour recommendation problem instances. Specifically, we use data from four main US cities, namely, New York City, Pittsburgh, San Francisco and Boston for a trip of 8 days, and we assume multiple user constraints. We expect that this setup is representative for several real-world cases. These results are reported in Section 5.2.

All methods described in this paper have been implemented in C\#. All the computational experiments are conducted on a Mac Pro equipped with 3.00 GHz Xeon CPU with 16 GB RAM and running Windows 10.

\subsection{Computational results on benchmark datasets for the MuPOPTW}

The dataset of Tricoire et al. (2010) consists of problem instances generated from a real-life industry application for software sales. They use the 

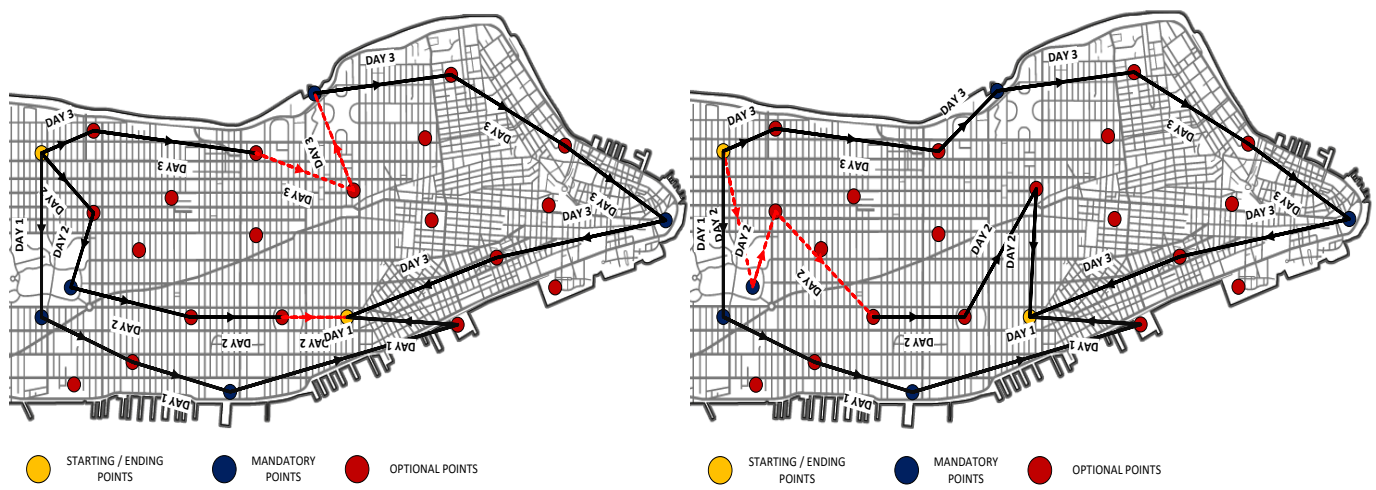

Figure 3: Inter-period relocate move

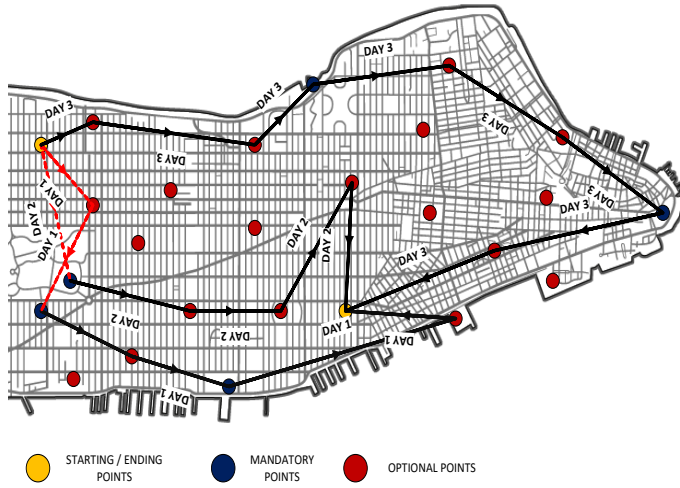

Figure 4: Intra-period relocate move

Figure 5: Inter-period exchange move

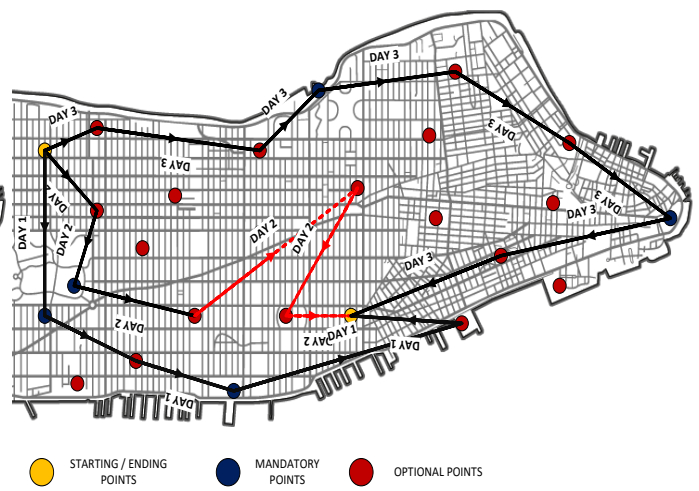

Figure 6: Intra-period exchange move

term 'customers' to represent nodes in their instances. In accordance with their notation, we use 'customer' and 'node' interchangeably in this section. Their instances include mandatory and optional nodes, multiple periods, node categories and service times according to the categories and multiple time windows for each node. More specifically, their instances include 6, 8, 10 or 12 mandatory nodes as well as 10, 20, 40, 80 or 140 optional nodes, a planning horizon of 3 days, 5 categories and 1, 2 or 3 time windows for each node.

The following parameter settings are used to perform the experiments for this dataset (a total of 1500 problem instances). The tabu list size $\delta$ is equal to 40. The probability for selecting points to remove $\gamma$ is 0.5 . The number of (internal) local search iterations without observing any improvement $\lambda_{\max }$ is set equal to 10000 , while the maximum number of perturbations $\vartheta_{\max }$ is 
equal to 10. Lastly, the number of restarts $\psi_{\max }$ is equal to 50. For each problem instance 10 simulation runs (occupying a single core) are performed and the best solution is reported.

Table 1 presents the results obtained on the benchmark datasets of Tricoire et al. (2010) for the MuPOPTW. Each row groups the problem instances with respect to the number of optional nodes (each group refers to 150 problem instances). The columns summarize the average results obtained by our algorithm (ITS), the average results reported by Tricoire et al. (2010) (VNS), the percentage gap (i.e. $=(\mathrm{ITS}-\mathrm{VNS}) /(\mathrm{VNS}) * 100)$ between the two methods (Gap \%) and the computational times (CPU Time) in seconds. The last row provides the average results for all problem instances. Note that the objective is to maximize the total collected profit.

Table 1: Overall comparison with Tricoire et al. (2010) VNS algorithm

\begin{tabular}{c|ccc|ccc|c}
\hline & \multicolumn{3}{|c}{ ITS } & \multicolumn{2}{c}{ VNS } & \multicolumn{2}{c}{ Gap } \\
\# Optional Points & Best Profit & Average Profit & CPU Time & Best Profit & CPU Time & $\%$ \\
\hline 10 & 38598.31 & 38598.31 & 0.21 & 38598.31 & 1.67 & 0.00 \\
20 & 44363.38 & 44363.38 & 0.48 & 44323.63 & 5.18 & 0.08 \\
40 & 47178.99 & 47161.31 & 14.77 & 47037.70 & 24.71 & 0.30 \\
80 & 51280.89 & 51257.06 & 1045.93 & 51389.43 & 143.96 & -0.21 \\
140 & 67471.41 & 67437.60 & 1451.69 & 67455.75 & 672.60 & 0.02 \\
\hline Average & 49778.60 & 49763.53 & 502.52 & 49760.97 & 169.62 & 0.04 \\
\hline
\end{tabular}

Overall, we can observe that our algorithm matches and improves (up to $0.3 \%$ on average) the previous results obtained by Tricoire et al. (2010) for all groups of problems except one (worst case performance is $-0.21 \%$ ). It also seems that our algorithm performs slightly better as the number of optional points increases; however, this is made at the expense of computational time. It is quite difficult to compare different approaches using different hardware and different number of simulation runs. It is also worth highlighting that with different parameter settings, for individual problem instances better solutions can be obtained. However, we select those settings that overall produce good results for each group. Lastly, we note the robustness of our algorithm, given that very small variations are observed between the best and the average results obtained for each set of runs.

\subsection{Real datasets from major US cities}

In this section, we present our experiments to evaluate the FFTS framework using a real dataset collected from Foursquare. 
The Foursquare dataset: We obtain an initial set of venues using the publicly available dataset of Cheng et al. (2011), which includes geo-tagged, user-generated content that was pushed to Twitter's public feed from September 2010 to January 2011. From this dataset, we select tweets related to Foursquare check-ins into venues with at least 10 check-ins. To that end, our final dataset consists of 6, 699, 516 check-ins.

Foursquare provides a classification of venues into types. This classification is hierarchical in the sense that a venue of type "Italian restaurant", belongs to the category "Restaurant", which further belongs to the higher level category "Food". At the time of data collection there were in total 9 top-level categories, namely, Arts \& Entertainment, College \& University, Food, Professional 86 Other Places, Nightlife Spots, Great Outdoors, Shop 66 Service, Travel 8 Transport and Residence. The original dataset did not include the category information for the venues, so we did the mapping of venues to types by crawling Foursquare. com.

For the purposes of our study, not all Foursquare venues form points that a visitor would be interested in. As a simple example, venues for hospitals and public services are included in the original dataset. Clearly such types of venues cannot be deemed as touristic points and we should not consider them in our experiments. Therefore, we first manually choose the lowest level types of venues in Foursquare that can be considered as points (either in $\mathcal{M}$ or $\mathcal{O}$ ) for our purposes. In particular, we pick 66 types - out of the 389 found in our dataset (the details are provided in the Appendix), and group them under 6 major categories (the details can be seen at Table 3).

Further, Foursquare provides the coordinates (latitude and longitude) of each point. The distance matrix between the points is constructed using the Haversine formula (Sinnott, 1984), while a unified average walking speed of 4 miles per hour is assumed for traversing each arc of the network.

We focus on 4 cities, namely, NYC, Pittsburgh, San Francisco and Boston. In particular, from our original Foursquare dataset we filter the check-ins in point type venues that were generated within a 20 miles radius from the city centers of the aforementioned cities as these were returned from the Google Maps API. Using each one of these 4 city datasets we further create inputs of mandatory points $\mathcal{M}$ of different sizes $|\mathcal{M}|(|\mathcal{M}| \in$ $\{5,10,20,40\})$ using random sampling. While there are many ways to compute the sampling probabilities, we utilize the popularity of a venue as 
captured through the number of its check-ins ${ }^{2}$. Once set $\mathcal{M}$ is chosen, set $\mathcal{O}$ is formed using our collaborative filtering method.

Collaborative Filtering: We use the open source Suggest (n.d.) software for obtaining $\mathcal{O}$. In particular, the user-item matrix $\Pi$ represents checkin information, while we use the similarity function (17). The value of $\alpha$ is computed using cross-validation. As alluded above when building the similarity model we use the $k$ most similar items for any given item. In our experiments, we set $k=20$. Furthermore, for every set $\mathcal{M}$, our CF method outputs $N=50$ recommended optional points, i.e., $|\mathcal{O}|=50$. Table 2 presents the number of items (i.e., points), users and transactions that are included in every city dataset. This table essentially encodes information about the dimension and sparsity of $\Pi$.

Table 2: Statistics for the NYC, SF, Pittsburgh and Boston User-Item Matrices.

\begin{tabular}{lrrrr}
\hline & NYC & SF & Pittsburgh & Boston \\
\hline \# Users & 11105 & 5076 & 707 & 2634 \\
\# Items & 2649 & 886 & 227 & 500 \\
\# Transactions & 59315 & 19478 & 2305 & 9529 \\
\hline
\end{tabular}

While generating the instances, we assign category based characteristics for profits, costs and service time constraints. Table 3 shows these characteristics with category information. While calculating the solutions, we also make the following assumptions:

- The trip needs to be completed in 8 days, i.e. $N_{p}=8$.

- The points are grouped under 6 categories, i.e. $N_{C}=6$. The category specific information can be seen at Table 3 .

- The category quotas for each category is 5 , i.e. $\phi_{k}^{p}=5 \quad \forall k \in C$.

- The maximum number of time windows for each point is 3, i.e. $N_{t}=3$. However, points might have 0 (not available),1,2, or 3 time windows depending on the day, time and category of the points.

\footnotetext{
${ }^{2}$ We also impose a loose spatial constraint to avoid spatially disparate sets of points. In particular, every new venue sampled is added to $\mathcal{M}$ if the average distance to all the current mandatory points is less than 10 miles; otherwise it is disregarded and a new venue is sampled.
} 
- 12 hours are devoted for each day of the trip, i.e. $D_{p}=12$.

- Each daily tour starts at 8 am and ends at 8pm.

- The starting and ending points of the daily tours are the same, i.e. $i_{0}^{1}=i_{0}^{2}=\ldots=i_{0}^{p}$ and $i_{n+1}^{1}=i_{n+1}^{2}=\ldots=i_{n+1}^{p}$

- The daily budget allocated for the points is $\$ 250$, i.e. $F_{p}=250$.

Furthermore, time windows of the points are determined from the checkin information from Foursquare. For each point, the peak check-in times (either 1, 2 or 3 different time frames) are obtained. The time windows are constructed so that a time window opens 1 hour before the peak check-in time and closes 1 hour after. Overlapping time windows are combined into one. If a time window is outside of the tour duration, i.e. from $8 \mathrm{am}$ to $8 \mathrm{pm}$, that point becomes unavailable. For the mandatory points, if the time windows are outside of the tour duration, their time windows are relaxed so that the points are available throughout the tour.

Table 3: Category information and category based point characteristics

\begin{tabular}{cccccc}
\hline Category & CF Category & Cat. Explanation & Service Time (h) & Cost (\$) & Profit \\
\hline 1 & A & Parks & 0.5 & 5 & 5 \\
2 & $\mathrm{~B}$ & Bars and Night Clubs & 1 & 10 & 10 \\
3 & $\mathrm{C}$ & Museums and Galleries & 2 & 15 & 15 \\
4 & $\mathrm{~N}$ & Great Outdoors & 0.5 & 10 & 10 \\
5 & $\mathrm{O}$ & Scenic Attractions & 0.5 & 15 & 15 \\
6 & $\mathrm{~T}$ & Movie and Broadway Theaters & 2 & 5 & 5 \\
\hline
\end{tabular}

We apply our algorithm to solve the resulting MP-MC-OP-MTW instances for each city and obtain the results for all generated $C F$ problem instances. For each city, 100 instances with randomly selected mandatory points are constructed. For the ITS algorithm, we adopted the same parameter settings as those described in Section 5.1; however, only one simulation run is performed for each instance.

Table 4 presents the overall results obtained from the Foursquare dataset. Each row groups the problem instances with respect to the number of mandatory points $(|\mathcal{M}|)$. The last row provides the average results. We present the overall profit (Profit) and computational time (CPU Time) in seconds for each problem group. Note that as the number of mandatory points increases, the complexity of the problem and computational times also increase, while more computational time is devoted for performing feasibility checks. 
Table 4: Results for $C F$ generated instances

\begin{tabular}{ccc}
\hline$|\mathcal{M}|$ & Profit & CPU Time \\
\hline 5 & 582.50 & 426.68 \\
10 & 621.25 & 530.66 \\
20 & 658.75 & 2431.80 \\
40 & 651.25 & 3675.53 \\
\hline Average & 628.44 & 1766.17 \\
\hline
\end{tabular}

Next, Table 5 presents a more detailed breakdown of the results from the Foursquare dataset. The problem instances are grouped in each row as per the number of mandatory points. The last row provides the average results for all problem instances. For each problem group, we present the average number of optional points included in the solutions (\# Optional Points Covered) and the overall percentage of optional points included (\% Optional Points Covered). Note that as the number of mandatory points increases, the number of optional points decreases, while keeping the overall number of points included roughly the same. We observe that feasibly inserting more optional points becomes more difficult as the number of mandatory points increases.

Table 5: Optional points covered for $C F$ generated instances

\begin{tabular}{ccc}
\hline$|\mathcal{M}|$ & \# Optional Points Covered & \% Optional Points Covered \\
\hline 5 & 47.25 & 90.86 \\
10 & 44.50 & 85.57 \\
20 & 40.5 & 77.88 \\
40 & 20 & 38.46 \\
\hline Average & 38.06 & 73.20 \\
\hline
\end{tabular}

Finally, Table 6 presents the distribution of the time budget between traveling, service and waiting times in hours. For each group of problems, the total transportation time needed (Transport Time), the total time used for service (Service Time) and the total time used for waiting for the point to become available (Waiting Time). The corresponding percentages with respect to the overall trip duration (96 hours) is reported (\%) next to each main column. The last row provides the average results for all problem instances. We believe the use of time budget for the trip can be a distinctive performance metric, since it has been used to evaluate the performance of orienteering algorithms in the literature. Note that the objective of maximizing the profit can only be possible by maximizing the points to be visited 
and spending most of the time budget on service, while keeping traveling and waiting times as low as possible. Overall, only $4.25 \%$ of the time budget is spent in waiting, while $58.76 \%$ is used for collecting profits by visiting points. These results are in line with our goal of providing high quality solutions for real life applications. Also observe that as the number of mandatory points increases, less time is spent for waiting, which is an indication of how compact the daily tours are becoming.

Table 6: Use of time budget for $C F$ generated instances

\begin{tabular}{cccc}
\hline \# Mandatory & Transport Time & Service Time & Waiting Time \\
\hline 5 & $35.84(37.33 \%)$ & $54.00(56.25 \%)$ & $6.16(6.41 \%)$ \\
10 & $37.10(38.65 \%)$ & $54.50(56.77 \%)$ & $4.40(4.58 \%)$ \\
20 & $34.58(36.03 \%)$ & $58.50(60.93 \%)$ & $2.91(3.03 \%)$ \\
40 & $34.51(35.94 \%)$ & $58.63(61.07 \%)$ & $2.86(2.98 \%)$ \\
\hline Average & $35.51(36.99 \%)$ & $56.41(58.76 \%)$ & $4.08(4.25 \%)$ \\
\hline
\end{tabular}

\section{Directions for real-world applications}

As the reliability of user generated content on tourism attractions is increasing, online applications are becoming an important alternative to paper-based guides. Among others, Google Trips (https://get.google. com/trips/) (Arsintescu et al., 2016), Visitacity (www.visitacity.com/), Triphobo (https://www .triphobo.com) and Sygic Travel (https://travel. sygic.com/) are popular examples of map-based applications that provide various trip planning and management functionalities, up-to-date tour recommendations and multiple itineraries to choose from. Even though they are easily accessible and increasingly popular, these applications fall short on providing personalized recommendations. Specifically, the existing online applications only provide a set of well-known attractions and pre-populated itineraries. Furthermore, the existing algorithms fail to recommend additional optional attractions apart from the ones that were initially selected by the user. In return, our proposed framework complements these existing online approaches in two major ways. First, our algorithm uses a small set of points that were given by the user to generate a bigger set of optional points. Second, our algorithm calculates the tours that make best use of time and maximize the utility for the user. The tours are created after the user inputs are finalized, as opposed to asking the user to select and alter a pre-populated tour for each day. 
The filtering component of our framework relies on user generated content from social media to create personalized trip recommendations. Specifically, our content is the check-ins and comments made using the Foursquare application. User generated content in the form of user reviews is very important in hospitality and tourism industry, since they play a significant role in shaping technology savvy tourists' opinions on certain hotels, restaurants and various other attractions (Ye et al., 2011, Ayeh et al., 2013). Using online web data allows the content to be updated in real time, thus it is more flexible than using published and offline media. To add, a tour can only be effective if all points included actually exist with accurate characteristics. For instance, if a tour recommender includes a point that is temporarily or permanently closed, it would impact the user satisfaction.

In addition to the value delivered to the users, our algorithm can be also beneficial for the promotion of tourism destinations. First, the success of regional tourism offices (RTO) depends on their abilities to engage in and respond to the community interests (Valente et al, 2015). Accordingly, our framework can allow RTOs to respond directly to the personalized interests of the tourists, and provide high quality valued services. To add, another important success measure of the RTOs is the rate of the returning tourists (Jarvis et al., 2016), which is highly correlated with tourist satisfaction (Tseng, 2017). On the other hand, RTOs can also possibly use a personalized tour recommendation tool for promoting specific events.

Lastly, trip planning and recommendations can be used to design itineraries that target specific market segments, and can be used for targeted marketing campaigns. Currently, destination marketing organizations (DMO) mainly use hotlines, brochures and advertisements to promote their destinations (Bansal and Eiselt, 2004). However, these strategies fall short in capturing the personal tastes of tourists. In return, personalized marketing communications promote the destination by user specific content, and increase the tourist attention to the area (Xiang and Gretzel, 2010). If the marketing costs are considered, personalized marketing communications are shown to be cheaper and more effective, compared to conventional destination marketing methods.

\section{Conclusions}

In this paper we introduced a multi-period orienteering problem with multiple time windows that was used to map a tour recommendation problem 
with personalized constraints and preferences. The objective is to maximize the personalized utility that is dynamically calculated based on information from social media and other online data sources. In an effort to capture the tourist's actual experience, our model considers many features and multiple user constraints, including among others visit restrictions and priorities, period dependent availabilities, duration and budget constraints, and upper bounds on the number point categories visited daily. We developed a FFTS framework that uses Collaborative Filtering for constructing the sets of mandatory and optional points to visit based on the user input, online reviews and the popularity of each point on social media, while an Iterated Tabu Search algorithm is employed to generate the daily tours.

We performed various experiments to assess and validate our framework in comparison with other state-of-the-art algorithms. Our results clearly show that our algorithm is competitive and produced various new best heuristic solutions on benchmark datasets of the literature. Furthermore, we generated a real-world dataset for four major US cities, using data collected from Foursquare and provided tour recommendations under various user constraints. Our results show that the proposed FFTS framework is robust and useful for solving tour recommendation problems.

We believe that the proposed FFTS framework can be of great interest in the tourism industry and it permits more flexible searching for attractions using social media as opposed to paper based guides. It can complement in various ways the existing online applications and personal navigation systems, while it can be used by RTOs and DMOs to offer additional added value services, to promote specific events and to create targeted marketing campaigns. Finally, worth pursuing research directions are to include multiple-modes of transportation with time-dependent travel times and costs as well as to consider multiple alternative objectives and present to the user all pareto solutions.

\section{References}

Anacleto, R., Figueiredo, L., Almeida, A., Novais, P. (2014). Mobile application to provide personalized sightseeing tours. Journal of Network and Computer Applications, 41, 56-64. http://dx.doi.org/10.1016/j.jnca. 2013.10.005 
Arsintescu, B., Gollapudi, S., Kollias, K., Sarlos, T., Tomkins, A. The 280 Year Old Algorithm Inside Google Trips [Blog post]. Retrieved from https://research.googleblog.com/2016/09/ the-280-year-old-algorithm-inside.html

Ayeh, J. K., Au, N., Law, R. (2013). Predicting the intention to use consumer-generated media for travel planning. Tourism Management, 35, 132-143. http://dx.doi.org/10.1016/j.tourman.2012.06.010

Bansal, H., Eiselt, H. A. (2004). Exploratory research of tourist motivations and planning. Tourism Management, 25(3), 387-396. http://dx.doi .org/ 10.1016/S0261-5177(03) 00135-3

Brilhante, I. R., Macedo, J. A., Nardini, F. M., Perego, R., Renso, C. (2015). On planning sightseeing tours with TripBuilder. Information Processing \& Management, 51(2), 1-15. http://dx.doi.org/10.1016/j.ipm. 2014. 10.003

Chao, I. M., Golden, B. L., Wasil, E. A. (1996). A fast and effective heuristic for the orienteering problem. European Journal of Operational Research, 88(3), 475-489. http://dx .doi .org/10.1016/0377-2217(95)00035-6

Cheng, Z., Caverlee, J., Lee, K., Sui, D. Z. (2011). Exploring millions of footprints in location sharing services. Proceedings of the Fifth International AAAI Conference on Weblogs and Social Media, 2011, 81-88. http: //www . aaai.org/ocs/index.php/ICWSM/ICWSM11/paper/viewFile/2783/3292

De Choudhury, M., Feldman, M., Amer-Yahia, S., Golbandi, N., Lempel, R., Yu, C. (2010, June). Automatic construction of travel itineraries using social breadcrumbs. In Proceedings of the 21st ACM conference on Hypertext and hypermedia (pp. 35-44). ACM. http://dx.doi.org/10.1145/ 1810617.1810626

Deshpande, M., Karypis, G. (2004). Item-based top-n recommendation algorithms. ACM Transactions on Information Systems (TOIS), 22(1), 143177. http://dx.doi.org/10.1145/963770.963776

Divsalar, A., Vansteenwegen, P., Sörensen, K., Cattrysse D. (2014). A memetic algorithm for the orienteering problem with hotel selection. European Journal of Operational Research 237, 29-49. http://dx.doi.org/ $10.1016 /$ j.ejor. 2014.01 .001 
Divsalar, A., Vansteenwegen, P., Cattrysse, A. (2013). A variable neighborhood search method for the orienteering problem with hotel selection. International Journal of Production Economics 145(1), 150-160. http://dx.doi.org/10.1016/j.ijpe.2013.01.010

Dunstall, S., Horn, M. E., Kilby, P., Krishnamoorthy, M., Owens, B., Sier, D., Thiebaux, S. (2003). An automated itinerary planning system for holiday travel. textitInformation Technology and Tourism, 6(3), 195-210. http: //dx.doi.org/10.3727/1098305031436944

Garcia, A., Vansteenwegen, P., Arbelaitz, O., Souffriau, W., Linaza, M.T., (2013). Integrating public transportation in personalised electronic tourist guides. Computers EOperations Research 40, 758-774. http://dx.doi. org/10.1016/j.cor.2011.03.020

Gavalas, D., Kenteris, M., Konstantopoulos, C., Pantziou, G. (2011). Web application for recommending personalised mobile tourist routes. Software IET, 6(4), 313-322. http://dx.doi.org/10.1049/iet-sen.2011.0156

Gavalas, D., Konstantopoulos, C., Mastakas, K., Pantziou, G. (2014). A survey on algorithmic approaches for solving tourist trip design problems. Journal of Heuristics, 20(3), 291-328. http://dx.doi.org/10. $1007 /$ s10732-014-9242-5

Gavalas, D., Konstantopoulos, C., Mastakas, K., Pantziou, G., Vathis, N. (2015). Heuristics for the time dependent team orienteering problem: Application to tourist route planning. Computers \&6 Operations Research, 62, 36-50. http://dx.doi.org/10.1016/j.cor.2015.03.016

Gunawan, A., Lau, H. C., Vansteenwegen, P. (2016). Orienteering Problem: A Survey of Recent Variants, Solution Approaches and Applications. European Journal of Operational Research. http://dx.doi.org/10.1016/j . ejor.2016.04.059

Hyde, K. F., Lawson, R. (2003). The nature of independent travel. Journal of Travel Research, 42(1), 13-23.http://dx.doi.org/10.1177\% 2F0047287503253944

Jarvis, D., Stoeckl, N., Liu, H. B. (2016). The impact of economic, social and environmental factors on trip satisfaction and the likelihood of visitors 
returning. Tourism Management, 52,1-18. http://dx.doi.org/10.1016/ j.tourman.2015.06.003

Kim, J., Kim, H., Ryu, J. H. (2009, April). TripTip: a trip planning service with tag-based recommendation. In CHI'09 Extended Abstracts on Human Factors in Computing Systems (pp. 3467-3472). ACM. http://dx.doi. org/10.1145/1520340.1520504

Rodriguez, B., Molina, J., Pérez, F., Caballero, R. (2012). Interactive design of personalised tourism routes. Tourism Management, 33(4), 926-940. http://dx.doi.org/10.1016/j.tourman.2011.09.014

Roy, S. B., Das, G., Amer-Yahia, S., Yu, C. (2011, April). Interactive itinerary planning. In Data Engineering (ICDE), 2011 IEEE 27th International Conference on (pp. 15-26). IEEE. http://dx.doi.org/10.1109/ ICDE . 2011.5767920

Sinnott, R. W. (1984). Virtues of the Haversine. Sky and Telescope, 68(2), 158-159. http: //adsabs . harvard. edu/abs/1984S\%26T . . .68R. 158S

Souffriau, W., Vansteenwegen, P., Vertommen, J., Berghe, G. V., Oudheusden, D. V. (2008). A personalized tourist trip design algorithm for mobile tourist guides. Applied Artificial Intelligence, 22(10), 964-985. http://dx.doi.org/10.1080/08839510802379626

Souffriau, W., Vansteenwegen, P., Vanden Berghe, G., Van Oudheusden, D. (2013). The multiconstraint team orienteering problem with multiple time windows. Transportation Science, 47(1), 53-63. http://dx.doi.org/10. $1287 / \operatorname{trsc} .1110 .0377$

Suggest. Top-N Recommendation engine, Version 1, George Karypis, University of Minnesota. http://glaros.dtc.umn.edu/gkhome/suggest/ overview.

Tarantilis, C.D., Stavropoulou, F., Repoussis, P.P. (2013a). The capacitated team orienteering problem: A bi-level filter-and-fan method. European Journal of Operational Research, 224 (1), pp. 65-78. http://dx.doi .org/ $10.1016 /$ j.ejor. 2012.07 .032 
Tarantilis, C. D., Anagnostopoulou, A. K., Repoussis, P. P. (2013b). Adaptive path relinking for vehicle routing and scheduling problems with product returns. Transportation Science, 47(3), 356-379. http://dx.doi.org/ $10.1287 / \operatorname{trsc} .1120 .0439$

Tricoire, F., Romauch, M., Doerner, K. F., Hartl, R. F. (2010). Heuristics for the multi-period orienteering problem with multiple time windows. Computers and Operations Research, 37(2), 351-367. http://dx.doi.org/10. $1016 / j$. cor. 2009.05.012

Tseng, A. (2017). Why do online tourists need sellers' ratings? Exploration of the factors affecting regretful tourist e-satisfaction. Tourism Management, 59, 413-424. http://dx.doi.org/10.1016/j.tourman.2016.08.017

Valente, F., Dredge, D., Lohmann, G. (2015). Leadership and governance in regional tourism. Journal of Destination Marketing 83 Management, 4(2), 127-136. http://dx.doi.org/10.1016/j.jdmm.2015.03.005

Vansteenwegen, P., Van Oudheusden, D. (2007). The mobile tourist guide: an OR opportunity. OR Insight, 20(3), 21-27. http://dx.doi.org/10. 1057/ori. 2007.17

Vansteenwegen, P., Souffriau, W., Berghe, G. V., Van Oudheusden, D. (2009). Iterated local search for the team orienteering problem with time windows. Computers and Operations Research, 36(12), 3281-3290. http://dx.doi.org/10.1016/j.cor.2009.03.008

Vansteenwegen, P., Souffriau, W., Van Oudheusden, D. (2011a). The orienteering problem: A survey. European Journal of Operational Research, 209(1), 1-10. http://dx.doi.org/10.1016/j.ejor.2010.03.045

Vansteenwegen, P., Souffriau, W., Berghe, G. V., Van Oudheusden, D. (2011b). The City Trip Planner: An expert system for tourists. Expert Systems with Applications, 38(6), 6540-6546. http://dx.doi.org/10.1016/ j. eswa. 2010.11 .085

Xiang, Z., Gretzel, U. (2010). Role of social media in online travel information search. Tourism Management, 31(2), 179-188. http://dx.doi.org/10. $1016 / j$.tourman.2009.02.016 
Ye, Q., Law, R., Gu, B., Chen, W. (2011). The influence of user-generated content on traveler behavior: An empirical investigation on the effects of e-word-of-mouth to hotel online bookings. Computers in Human Behavior, 27(2), 634-639. http://dx.doi.org/10.1016/j.chb.2010.04.014

Zhu, C., Hu, J. Q., Wang, F., Xu, Y., Cao, R. (2012). On the tour planning problem. Annals of Operations Research, 192(1), 67-86. http://dx.doi. org/10.1007/s10479-010-0763-5

\section{Appendix A. Venue Types}

From the 389 different types of venues found in our dataset, we designate as points to visit the following categories: Skate Park, Hotel Bar, Theme Park, Planetarium, Rock Club, General Entertainment, Water Park, Ski Chalet, Sake Bar, Pub, Art Museum, Opera House, Harbor or Marina, Golf Course, Mountain, Ski Area, Hookah Bar, Whisky Bar, Cocktail Bar, Brewery, Beer Garden, Nightclub, Speakeasy, Multiplex, Hiking Trail, Bar, Museum, Scenic Lookout, Pier, Theater, Nightlife Spot, Art Gallery, Piano Bar, Music Venue, Indie Movie Theater, Other Great Outdoors, Surf Spot, Indie or Off Broadway Theater, Bridge, Gay Bar, History Museum, Cafeteria, Wine Bar, Arts 8 Entertainment, Shrine, Ski Lodge, Dive Bar, Great Outdoors, Lighthouse, Beach, Comedy Club, Garden, Winery, Apres Ski Bar, Hot Spring, Mosque, Jazz Club, Movie Theater, Monument or Landmark, Science Museum, Other Nightlife, Skating Rink, Lake, Sports Bar, Historic Site and Sculpture Garden. 